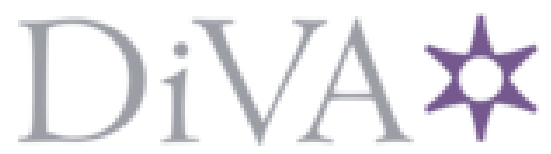

http://www.diva-portal.org

This is the published version of a paper published in Scandinavian Journal of Medicine and Science in Sports.

Citation for the original published paper (version of record):

Andersson, H M., Karlsen, A., Blomhoff, R., Raastad, T., Kadi, F. (2010)

Plasma antioxidant responses and oxidative stress following a soccer game in elite female players.

Scandinavian Journal of Medicine and Science in Sports, 20(4): 600-608

https://doi.org/10.1111/j.1600-0838.2009.00987.x

Access to the published version may require subscription.

N.B. When citing this work, cite the original published paper.

Permanent link to this version:

http://urn.kb.se/resolve?urn=urn:nbn:se:oru:diva-10875 


\title{
Plasma antioxidant responses and oxidative stress following a soccer game in elite female players
}

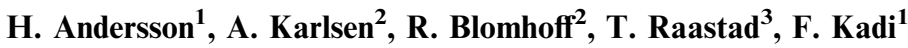 \\ ${ }^{1}$ School of Health and Medical Sciences, Örebro University, Örebro, Sweden, ${ }^{2}$ Department of Nutrition, Institute of Basic Medical \\ Sciences, University of Oslo, Oslo, Norway, ${ }^{3}$ Norwegian School of Sport Sciences, Oslo, Norway \\ Corresponding author: Fawzi Kadi, School of Health and Medical Sciences, Örebro University, 70182 Örebro, Sweden. Tel: \\ +461930 1160, Fax: +461930 3486,E-mail:fawzi.kadi@oru.se
}

Accepted for publication 18 May 2009

We aimed to investigate markers of oxidative stress and levels of endogenous and dietary antioxidants in 16 elite female soccer players in response to a 90-min game (average intensity $82 \pm 3 \%$ HRpeak). Blood samples were taken before, immediately and $21 \mathrm{~h}$ after the game. Plasmaoxidized glutathione, the ratio of reduced to oxidized glutathione (GSH:GSSG) and lipid peroxidation measured by d-ROMs were used as markers of oxidative stress. Plasma endogenous [uric acid, total glutathione (TGSH)] and dietary antioxidants $(\alpha$-tocopherol, ascorbic acid, total carotenoids and polyphenols) were analyzed using liquid chromatography and the Folin-Ciocalteu method. Exercise induced an acute increase $(P<0.05)$ in GSSG, uric acid,
TGSH, $\alpha$-tocopherol, and ascorbic acid. In parallel, the GSH:GSSG ratio and polyphenols decreased $(P<0.05)$. GSSG, GSH:GSSG ratio, uric acid, TGSH, and ascorbic acid returned to baseline at $21 \mathrm{~h}$, while polyphenols and $\alpha$-tocopherol remained altered. Total carotenoids increased above baseline only at $21 \mathrm{~h}(P<0.05)$. Lipid peroxidation, measured by d-ROMs, remained unchanged throughout the study. Thus, intermittent exercise in well-trained female athletes induces a transient increase in GSSG and a decrease in the GSH:GSSG ratio, which is effectively balanced by the recruitment of both endogenous and dietary antioxidants, resulting in the absence of lipid peroxidation measured by d-ROMs.
An exercise stimulus has been shown to induce the production of free radicals, which may lead to oxidative stress and cause damage to cellular tissue (Davies et al., 1982). During exercise, whole-body oxygen consumption may increase up to 20 -fold (Saltin \& Åstrand, 1967), while oxygen consumption in active muscles may reach 100 times the resting level (Davies et al., 1982). Most of the oxygen consumption in skeletal muscle is used to form ATP and water ( $\sim 95 \%$ ), while the remaining $2-5 \%$ undergoes a reduction to produce superoxide radicals (Jackson, 1998). Under normal physiological conditions, the quantity of reactive oxygen and nitrogen species (ROS/RNS) is in a fine-tuned equilibrium with the antioxidant defense system, consisting of endogenous and dietary antioxidant compounds. Imbalances may be mediated by increased ROS/RNS production or depletion in the antioxidant defense system. Imbalances in favor of increased ROS/RNS may be followed by oxidative damage. Oxidative damage to cellular targets is characterized by a progressive change or degradation of biomolecules as lipids, proteins, and DNA, and the recruitment of defense mechanisms may prevent or limit these changes (Djordjević, 2004; Blomhoff, 2005).
Exhaustive exercise has been shown to produce excessive amounts of ROS/RNS, leading to oxidative stress (Sastre et al., 1992). However, in well-trained athletes, only limited production of ROS/RNS may occur. This may be explained by a well-adapted antioxidant defense system in athletes (Brites et al., 1999). Whether a balance between the production of ROS/RNS and the recruitment of antioxidant defense systems prevents the occurrence of lipid peroxidation in response to exercise in well-trained athletes is, however, not fully understood. Moreover, most studies on oxidative stress and antioxidant responses to exercise have been performed using prolonged endurance exercise protocols (Aguiló et al., 2005; Tauler et al., 2005). In this respect, the physiological load of intermittent exercise, such as soccer games, differs from a continuous steady-state exercise. Consequently, extrapolation of data from a continuous steady-state exercise to intermittent exercise should be made with caution. During a soccer game, the aerobic energy system is highly taxed (Bangsbo, 1994; Krustrup et al., 2005) and a high load is exerted on active muscles (Andersson et al., 2008). In this respect, increases in markers of oxidative stress in plasma following soccer games and soccer-specific 


\section{Andersson et al.}

running protocols in male players have been suggested to occur (Kingsley et al., 2005; Ascensão et al., 2008; Ispirlidis et al., 2008; Tauler et al., 2008). Increased oxidative stress caused by training and games may compromise the exercise performance of players during a long season and especially during times with the most intensive match schedule (Ascensão et al., 2008). Understanding how oxidative stress and the antioxidant defense system respond following games in female players may therefore provide new information on the physiological effects of soccer games. It may also provide valuable information on the recovery processes following games, which is specifically important for women soccer players as there are only 2 days of recovery between games in international tournaments. Furthermore, it may also yield new information that can be used to scientifically address the issue related to the usage of antioxidant supplements by elite female players. Evidences in favor of increased antioxidant defense in plasma following soccer games in males have, in fact, been supported by some (Kingsley et al., 2005; Ascensão et al., 2008) but not all studies (Kingsley et al., 2005; Tauler et al., 2008). To our knowledge, the responses of antioxidant compounds and oxidative stress markers immediately and $21 \mathrm{~h}$ after an elite female soccer game have not been documented previously.

Given the above discussion, the aim of this study was to investigate the responses of plasma markers of oxidative stress [oxidized glutathione, ratio of reduced and oxidized glutathione (GSH:GSSG), lipid peroxidation measured by d-ROMs] and levels of endogenous [uric acid, total glutathione (TGSH)] and dietary (ascorbic acid, $\alpha$-tocopherol, total polyphenols, and total carotenoids) antioxidants immediately and $21 \mathrm{~h}$ after intermittent exercise consisting of an international 90-min friendly soccer game.

\section{Methods}

\section{Subjects}

Twenty-two elite female soccer players played an international 90 -min friendly game. The players participating in the study played in teams of the highest division in Sweden and Norway. Owing to economical restrictions, blood samples from 16 field players (height: $167 \pm 5 \mathrm{~cm}$, weight: $64 \pm 2 \mathrm{~kg}$, age: $22 \pm 3$ years, maximal oxygen consumption $54 \pm 3 \mathrm{~mL} / \mathrm{kg} / \mathrm{min}$ ) were randomly chosen for the analyses of markers of oxidative stress and antioxidant levels. The game was conducted in the middle of the soccer season, when the players are accustomed to playing games. Before the commencement of the study, the players had rested at least 3 days from games and 2 days from soccer training. The players were informed about the experimental procedures and possible discomforts associated with the study, and a written informed consent was obtained. The study was conducted according to the Declaration of Helsinki and approved by the Regional Ethic Committee of Uppsala, Sweden (pnr 2004: m-364).

\section{Experimental design}

Baseline values for oxidative stress markers and antioxidant levels were sampled at 10.00 hours, i.e., $1 \mathrm{~h}$ before the start of the soccer game (baseline). Blood samples were collected within 15-20 min $(0 \mathrm{~h})$ and $21 \mathrm{~h}$ (after breakfast at 10.00 hours the following morning) after the game. Plasma samples were analyzed for oxidized glutathione (GSSG) as a marker of oxidative stress, GSH:GSSG ratio as an indicator of redox status (Powers \& Jackson, 2008) and the d-ROMs test as a marker of lipid peroxidation (Cesarone et al., 1999). Additionally, two endogenous antioxidants (uric acid and TGSH) and several dietary antioxidants (ascorbic acid-AA-, $\alpha$-tocopherol, polyphenols and carotenoid compounds) were analyzed in plasma. We only measured two endogenous antioxidants in the present study. However, the activity of the enzymes superoxide dismutase (SOD), catalase (CAT), and glutathione peroxidase (GPx) are major consituents of the endogenous antioxidant defense system (Ji, 2000). Therefore, the term "endogenous defence system" in the present study only refers to the response of TGSH and uric acid. Two days before the commencement of the study the players performed a maximal oxygen uptake test running on a treadmill. Throughout the study period, the players were supervised in order to standardize the physical activities and diet.

\section{Diet}

The food intake was standardized for all players during the study period, starting on the evening before the game. All players were given a meal plan formulated by a nutritionist. The players ate each meal together. The players were given a dinner on the night before the game, breakfast before the game, lunch and dinner after the game and breakfast on the following morning. The composition of the meals was developed using a national food database ("Food on data" 4.3 LKH, Norway). Carbohydrate (CHO) and protein intake were adjusted according to the players' body weight $(55 / 60 / 65 / 70 \mathrm{~kg}$, respectively) to meet the recommendations for daily recovery (intake of $\geq 6 \mathrm{~g} / \mathrm{kg}$ body weight $\mathrm{CHO}$ and $\geq 1.2 \mathrm{~g} / \mathrm{kg}$ body weight protein) (Maughan et al., 2004). The food was chosen to replicate the player's normal diet as much as possible and did not contain any food items with known high antioxidant levels. The meal plan included a variation of bread, cereals, milk/ yoghurts, meat, pasta/rice, fruit, and vegetables to ensure adequate intake of macro- and micronutrients. In addition, the players were instructed to drink a sports drink during the game providing approximately $30-60 \mathrm{~g} \mathrm{CHO} / \mathrm{h}$ (Maxim En$\left.\mathrm{ergy}^{\mathrm{TM}}\right)$. The sports drink did not contain vitamin C. After the blood sample was taken at the end of the game, each player had a $\mathrm{CHO}$ intake of $1 \mathrm{~g} / \mathrm{kg}$ body weight within $30 \mathrm{~min}$ to ensure optimal recovery (banana, yoghurt, and sports drink) (Maughan et al., 2004).

\section{Evaluation of aerobic work load during the game}

Heart rate was recorded to evaluate the aerobic work load during the game. The players wore a heart rate monitor around their chest, and data were continuously collected every 5 s (Polar Team System, Polar Electro OY, Kempele, Finland).

\section{Biochemistry}

Blood samples were collected from the antecubital vein at all time points (baseline, immediately and $21 \mathrm{~h}$ post game). Uric acid was analyzed using standard routine measurements in a 
Modular $\mathrm{P}^{\mathbb{E}}$ Analyzer (Hitachi, Tokyo, Japan) with a CV of $<5 \%$. The Diacrons reactive oxygen metabolite (d-ROMs) test was used to assess lipid peroxidation and was performed according to the manufacturer's instructions (Diacron International, Grossetto, Italy). The d-ROMs test monitors a rather persistent radical cation formed in the reaction of alkoxy and peroxy radicals derived from the hydroperoxides with a suitable additive $N, N$,-diethyl-para-phenylendiamine (DEPPD). Thus, the d-ROMs level is proportional to the serum hydroperoxide concentration in the plasma (Alberti et al., 2000). Hydroperoxides are products of the peroxidation of proteins, peptides, amino acids, lipids and fatty acids (Nakayama et al., 2007). The analysis was fully automated using a Technicon RA 1000 system (Technicon Instruments Corporation, New York). The test was carried out in the kinetic mode. Results are expressed in arbitrary units (CARR Units) (Cesarone et al., 1999) with a CV $<10 \%$. According to the manufacturer's instructions, a normal value for d-ROMs is between 250 and 300 CARR $U$ in healthy individuals. In trained individuals, lower basal values have been reported ( $<250$ CARR U) (Banfi et al., 2006). A value between 301 and 320 CARR $U$ indicates a borderline condition of oxidative stress status, while a value above 320 CARR U indicates oxidative stress (Diacron International).

TGSH in the plasma was analyzed with the use of chemical reduction and according to the "homocysteine by HPLC" kit provided by Biorad Laboratories $\mathrm{GmbH}$ (Munchen, Germany). Standard solutions prepared in PBS served as calibrators with a $\mathrm{CV}<5 \%$. In comparison with whole-blood glutathione, plasma contains $<1 \%$ glutathione (Ji, 2000). However, plasma glutathione can be used as a model for intracellular glutathione, as it is assumed that plasma glutathione reflects intracellular whole-blood glutathione. Reduced plasma glutathione constitutes about $50-70 \%$ of the total plasma glutathione while GSSG constitutes about $5-10 \%$ of total glutathione. Moreover, the fraction of TGSH in our study includes components other than pure glutathione, such as adducts of plasma proteins, while GSH and GSSG is free plasma glutathione. Hence, the observed value of TGSH is higher compared with GSH and GSSG. Quantification of endogenous oxidized glutathione (GSSG) and reduced glutathione (GSH) was performed using a two-dimensional chromatographic system with parallel Hypercarb columns coupled with dual fluorescence detectors (FLD). After sample preparation as described previously (Sakhi et al., 2006), $10 \mu \mathrm{L}$ of the supernatant was injected into the chromatographic system. Derivatization of GSSG was performed using monobromobimane (MBB) and ortho-phthalaldehyde (OPA). The $\mathrm{CV}$ for the method was below 7\%. The ratio of GSH:GSSG was calculated by dividing GSH with GSSG.

For the determination of ascorbic acid in plasma, heparinized plasma was immediately acidified using an equal volume of $10 \%$ MPA and stored at $-70{ }^{\circ} \mathrm{C}$ until analysis within 3 months. Samples were analyzed by high-performance liquid chromatography (HPLC) (Karlsen et al., 2005, 2007) with a CV below $5 \%$. Plasma calibrators quantified against the NIST 970 SRM served as standards. For the determination of $\alpha$-tocopherol by HPLC, proteins were precipitated by the addition of 3 volumes of isopropanol, followed by centrifugation at $3000 \mathrm{~g}$ at $4{ }^{\circ} \mathrm{C}$ for $15 \mathrm{~min}$. The internal standard tocol was added with the isopropanol and $5 \mu \mathrm{L}$ of the clear supernatant was used for analysis (Richheimer et al., 1994). A fluorescence detector operated at 295 (ex) and 330 (em) was used for the detection with a CV below $5 \%$. Standards prepared in $1 \%$ bovine serum albumin in phosphate-buffered saline were used for quantification. For analysis of total polyphenols, $50 \mu \mathrm{L}$ heparinized plasma were mixed with $150 \mu \mathrm{L}$ ethanol for $2 \mathrm{~min}$, before centrifugation at
$3000 \mathrm{~g}$ at $4{ }^{\circ} \mathrm{C}$ for $15 \mathrm{~min}$. Fifty microliters of the clear supernatant was used for the Folin-Ciocalteu method as described previously (Maskarinec et al., 1999). Quercetin prepared in ethanol served as a standard solution, and the results for polyphenols are given as $\mu \mathrm{mol} / \mathrm{L}$ quercetin equivalents $(\mathrm{QE})$ with a $\mathrm{CV}$ below $10 \%$. The total carotenoid is the sum of lutein, zeaxanthin, $\beta$-crytpoxanthin, $\alpha$-carotene, $\beta$-carotene, and lycopene. They were determined in plasma by HPLC. Proteins were precipitated and removed by the addition of a 4.5 volume of isopropanol, followed by centrifugation at $3000 \mathrm{~g}$ at $4{ }^{\circ} \mathrm{C}$ for $15 \mathrm{~min}$. The internal standard astaxanthin was added in the isopropanol. Twenty-five microliters of the clear supernatant was used for analysis. The mobile phases consisted of A: $20 \%$ water and $24 \%$ acetone in ethanol and $\mathrm{B}$ : acetone. The gradient conditions were as follows: from $2 \%$ to $100 \% \mathrm{~B}$ within $20 \mathrm{~min}$, followed by $100 \%$ B for $15 \mathrm{~min}$. Detection was performed at $453 \mathrm{~nm}$ using a variable wavelength detector. Plasma calibrators quantified against the NIST 968c SRM were used as standards and the $\mathrm{CV}$ for the method was below $5 \%$.

\section{Statistical analysis}

A repeated-measure ANOVA for normally distributed data was used to test for possible significant time differences. When a significant time difference was identified, a Tukey's post hoc test was used. The d-ROMs data were not normally distributed, and were log-10 transformed before ANOVA. $P$-values below 0.05 were considered statistically significant. Data are presented as means and standard error of the mean (mean \pm SEM), except for heart rate data, which are reported as means and standard deviation (mean \pm SD). The Statistical Package for the Social Sciences (SPSS Inc., version 12.0) and Statistica (StatSoft Inc., version 7.0) were used for the analyses.

\section{Results}

Aerobic work load during the game

The average heart rate during the game was $163 \pm 2$ b.p.m., which corresponds to $82 \pm 3 \%$ of HRpeak. The players spent on average $40 \pm 4 \mathrm{~min}$ (Table 1) above $85 \%$ of HRpeak during the game, defined as a high-intensity work load (Bangsbo, 1994).

\section{Markers of oxidative stress and antioxidant levels immediately after the game}

The absolute GSSG concentration significantly increased immediately after the game $(30 \pm 9 \%$, $P<0.05)$. No changes were observed in GSH,

Table 1. Amount of time (min) spent in different heart rate zones during the soccer game

HR zone

(\% of HRpeak)

Total minutes (min) $3 \pm 113 \pm 235 \pm 225 \pm 213 \pm 32 \pm 1$

Data are presented as mean \pm SD . 
Andersson et al.

Table 2. The response of oxidative stress markers, endogenous, and dietary antioxidant levels following an elite female soccer game

\begin{tabular}{|c|c|c|c|}
\hline$n=16$ & Baseline values & Immediately post game & $21 \mathrm{~h}$ post game \\
\hline \multicolumn{4}{|l|}{ Oxidative stress markers } \\
\hline GSSG $(\mu \mathrm{mol} / \mathrm{L})$ & $0.047 \pm 0.003$ & $0.056 \pm 0.004^{*}$ & $0.051 \pm 0.003$ \\
\hline GSH:GSSG & $41.4 \pm 3.1$ & $35.1 \pm 2.9^{\#}$ & $37 \pm 2.7$ \\
\hline d-ROMs (CARR U) & $245 \pm 25$ & $249 \pm 25$ & $244 \pm 24$ \\
\hline \multicolumn{4}{|l|}{ Endogenous antioxidant levels } \\
\hline Uric acid $(\mu \mathrm{mol} / \mathrm{L})$ & $251 \pm 14$ & $277 \pm 15^{\star}$ & $254 \pm 14$ \\
\hline $\mathrm{TGSH}(\mu \mathrm{mol} / \mathrm{L})$ & $3.5 \pm 0.1$ & $4.3 \pm 0.2^{*}$ & $3.9 \pm 0.1$ \\
\hline $\mathrm{GSH}(\mu \mathrm{mol} / \mathrm{L})^{\prime}$ & $1.9 \pm 0.1$ & $2.0 \pm 0.2$ & $2.0 \pm 0.2$ \\
\hline \multicolumn{4}{|l|}{ Dietary antioxidant levels } \\
\hline Total polyphenols $(\mu \mathrm{mol} / \mathrm{L} Q \mathrm{QE})$ & $332 \pm 10$ & $264 \pm 4^{\#}$ & $245 \pm 12^{\#}$ \\
\hline Ascorbic Acid $(\mu \mathrm{mol} / \mathrm{L})$ & $54 \pm 3$ & $70 \pm 4^{*}$ & $58 \pm 3$ \\
\hline$\alpha$-topcopherol $(\mu \mathrm{mol} / \mathrm{L})$ & $20 \pm 1$ & $23 \pm 1^{\star}$ & $22 \pm 1^{\star}$ \\
\hline Lutein $(\mu \mathrm{mol} / \mathrm{L})$ & $0.18 \pm 0.02$ & $0.19 \pm 0.02$ & $0.23 \pm 0.03^{*}$ \\
\hline Zeaxanthin $(\mu \mathrm{mol} / \mathrm{L})$ & $0.04 \pm 0.00$ & $0.04 \pm 0.00$ & $0.06 \pm 0.01^{*}$ \\
\hline$\beta$-crytpoxanthin $(\mu \mathrm{mol} / \mathrm{L})$ & $0.18 \pm 0.03$ & $0.19 \pm 0.04$ & $0.23 \pm 0.06^{*}$ \\
\hline$\alpha$-carotene $(\mu \mathrm{mol} / \mathrm{L})$ & $0.13 \pm 0.02$ & $0.12 \pm 0.02$ & $0.15 \pm 0.04$ \\
\hline$\beta$-carotene $(\mu \mathrm{mol} / \mathrm{L})$ & $0.51 \pm 0.06$ & $0.49 \pm 0.06$ & $0.58 \pm 0.10$ \\
\hline Lycopene $(\mu \mathrm{mol} / \mathrm{L})$ & $0.56 \pm 0.04$ & $0.53 \pm 0.04$ & $0.65 \pm 0.08$ \\
\hline Total carotenoids ( $\mu \mathrm{mol} / \mathrm{L})$ & $1.6 \pm 0.1$ & $1.6 \pm 0.1$ & $1.9 \pm 0.3^{\star}$ \\
\hline
\end{tabular}

Data are presented as mean $\pm \mathrm{SE}$.

$P<0.05$.

${ }^{\star}$ Significantly higher and

\#Significantly lower compared with baseline values.

whereas the GSH:GSSG ratio decreased $(13 \pm 7 \%$, $P<0.05)$. No changes in d-ROMs levels were detected after the game (Table 2). This indicates that oxidized glutathione increases, and GSH:GSSG decreases immediately after the game without increases in the peroxidation levels measured by d-ROMs. The antioxidants TGSH $(23 \pm 7 \%)$, uric acid $(11 \pm 2 \%)$, AA $(33 \pm 7 \%)$, and $\alpha$-tocopherol $(15 \pm 2 \%)$ significantly increased immediately after the game whereas total polyphenols significantly decreased $(18 \pm 3 \%)$ (Table 2). In contrast, the carotenoid compounds (lutein, zeaxanthin, $\beta$-crytpoxanthin, $\alpha$-carotene, $\beta$-carotene, and lycopene) remained unchanged from baseline values (Table 2). This indicates an acute recruitment of both endogenous antioxidants and the majority of dietary antioxidants after the game.

\section{Markers of oxidative stress and antioxidant levels at $21 \mathrm{~h}$}

The GSSG concentration and GSH:GSSG ratio returned to baseline levels at $21 \mathrm{~h}$ [Fig. 1(a)]. The dROMs levels remained unchanged. The endogenous antioxidant compounds uric acid and TGSH and the dietary antioxidant AA returned to baseline levels $21 \mathrm{~h}$ after the game [Fig. 1(b) and (c)]. In contrast, the total polyphenols remained significantly reduced whereas $\alpha$-tocopherol remained elevated [Fig. 1(b)]. Total carotenoids significantly increased above baseline levels only at $21 \mathrm{~h}$ after the game, mainly due to increases in lutein, zeaxanthin and $\beta$-crytpoxanthin (Table 2).

\section{Discussion}

The present study shows for the first time that $90 \mathrm{~min}$ of intermittent exercise in well-trained female soccer players induces a mobilization of both endogenous and dietary antioxidants in response to a transient increase in GSSG and a decrease in the GSH:GSSG ratio. The recruitment of the antioxidant defense seemed to prevent lipid peroxidation measured by d-ROMs. Furthermore, our results indicate that the dietary antioxidant polyphenols may play an important role in the dietary antioxidant defense during intermittent exercise.

Changes in plasma thiols, especially oxidized glutathione and GSH:GSSG ratio, have been used as markers of oxidative stress status in biological systems (Ji, 1999). Hence, the significant increase in GSSG and decrease in the GSH:GSSG ratio observed immediately after the game indicate increased free-radical production. Considering that $2-5 \%$ of the total $\dot{V O}_{2}$ results in the formation of ROS/RNS (Jackson, 1998) and that the players worked at an average intensity level of $82 \%$ of HRpeak (approximately $67 \%$ of $\dot{V O}_{2}$ peak), an increased production of ROS/RNS was expected during the soccer game. The observed increases in GSSG and decrease in the GSH:GSSG ratio are in agreement with data 

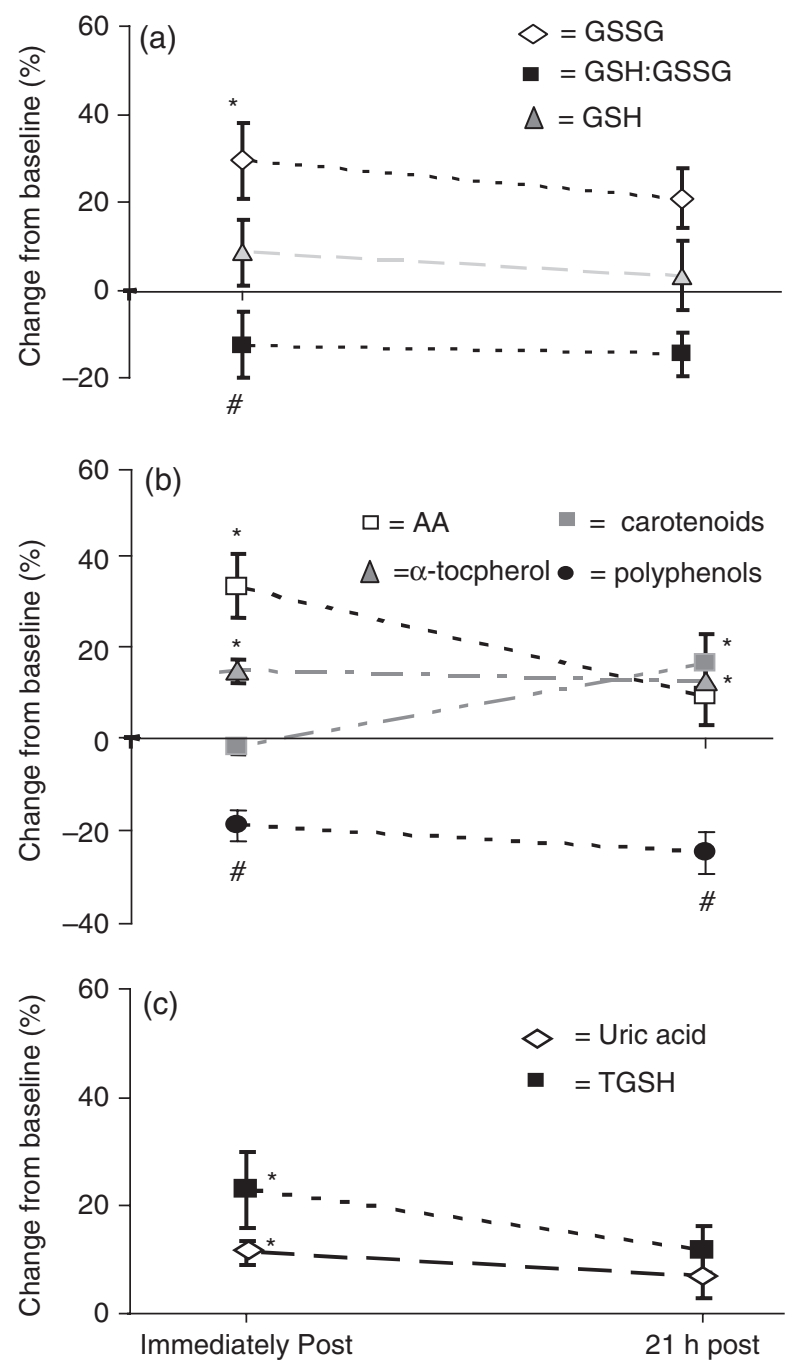

Fig. 1. Changes in (a) GSSG, GSH:GSSG and GSH (b) dietary antioxidants (AA, $\alpha$-tocopherol, carotenoids and polyphenols), and (c) endogenous antioxidants [uric acid and total glutathione (TGSH)] immediately post and $21 \mathrm{~h}$ after the soccer game. Data represent percentage change from baseline values. *Significantly higher than baseline values, $P<0.05$. \#Significantly lower than baseline values, $P<0.05$.

reported during various exercise protocols (Viguie et al., 1993; Chung et al., 1999; Watson et al., 2005). The changes in the GSSG levels and GSH:GSSG ratio were brief and returned to baseline within $21 \mathrm{~h}$ following the game. In parallel with the increase in GSSG and decreased GSH:GSSG ratio, an immediate mobilization of the antioxidant defense system occurred. To our knowledge, we report for the first time a significant decrease in total polyphenols immediately after exercise. This suggests that plasma polyphenols are instantly utilized to eliminate the increased levels of ROS/RNS. Polyphenols represent a large group of aromatic compounds and exist in most food items. Studies on polyphenols suggest that they have powerful antioxidant activities and are able to scavenge a diversity of ROS species or even inhibit their formation (Halliwell et al., 2005; Morillas-Ruiz et al., 2006). Polyphenols are available in the circulation but are not known to be stored in peripheral tissues (Rechner et al., 2002). Based on our results, it is reasonable to suggest that polyphenols are part of the first line of defense against oxidative stress in well-trained athletes. However, the question of whether polyphenols are mobilized in sedentary subjects following intermittent exercise remains unknown.

In parallel with the decrease in polyphenols, we observed an increase in the endogenous antioxidant compounds uric acid and TGSH. Similar changes in uric acid as well as the total antioxidant status have been shown after soccer games in male players (Bangsbo, 1994; Ascensão et al., 2008). Elevated levels of blood antioxidants at the end of an exercise bout may be explained by either reduced oxidant production at the end of the exercise, up-regulation of endogenous antioxidants and/or the mobilization of antioxidants from tissue stores as a result of increased free-radical production during exercise (Watson et al., 2005). As the up-regulation of uric acid and TGSH occurred immediately after the game, these endogenous antioxidants seem to play important roles in the antioxidant defense during the acute phase following intermittent exercise. Uric acid functions as an antioxidant due to its free radicalquenching actions in plasma as well as in skeletal muscle (Hellsten et al., 1997). It has been suggested that the contribution of uric acid in the total plasma antioxidant capacity is approximately $35-65 \%$ (Wayner et al., 1987), indicating its importance in the antioxidant defense system. Increased plasma levels of glutathione during exercise have been proposed to originate from the liver, as liver glutathione is shown to decrease during exercise (Sen et al., 1992; Leeuwenburgh \& Ji, 1996). The release of glutathione from the liver during exercise may be promoted by increased levels of catecholamines, glucagon, and vasopressin (Ji, 2000). As an antioxidant, glutathione can directly detoxify ROS, serves as a substrate for glutaredoxin to eliminate $\mathrm{H}_{2} \mathrm{O}_{2}$ and organic hydroperoxides and enhance the functional ability of other crucial antioxidants such as vitamin E and C (Sen \& Packer, 2000). The TGSH fraction measured in our study also includes other components such as adducts of plasma proteins. This explains the fact that the concentration of TGSH is higher than GSSG.

The increased levels of the dietary antioxidants, $\alpha$-tocopherol and AA observed immediately after the game suggest mobilization of tissue antioxidants into the circulation. The increase in AA is similar to what has been reported previously following $90-\mathrm{min}$ soccer-specific intermittent running protocols in male subjects (Thompson et al., 2001; Kingsley et al., 


\section{Andersson et al.}

2005). It has been suggested that the contribution of AA and $\alpha$-tocopherol to the total antioxidant capacity in plasma is about $0-24 \%$ and $5-10 \%$, respectively (Wayner et al., 1987). Unlike our results, no changes were observed in plasma ascorbate and $\alpha$ tocopherol immediately after a 60 -min game in male players (Tauler et al., 2008). The observation of unchanged total carotenoids immediately after the game is in agreement with previous studies using male subjects after a soccer game and endurance biking (Aguiló et al., 2005; Tauler et al., 2005, 2008), and is likely explained by the slower release of carotenoids from their main storage in adipose tissue. Nevertheless, the contribution of carotenoids to the total antioxidant capacity pool is relatively unknown (Watson et al., 2005).

Interestingly, no significant changes were observed in the d-ROM levels immediately or $21 \mathrm{~h}$ after the game. Harma et al. (2006) have questioned the specificity and sensitivity of the d-ROMs test to measure oxidative stress in sports medicine (Harma et al., 2006). More specifically, it has been argued that the d-ROMs test detects ferroxidase activity (Erel, 2005). However, the relationship between d-ROMs test and ferroxidase activity has initially been described by the manufacturer. Indeed, Alberti et al. (2000) reported that the contribution of ceruloplasmin ferroxidase activity is small. First, it has been demonstrated that the oxidase activity of ceruloplasmin is diluted when the serum sample undergoes a 100-fold dilution, which is the case in the d-ROMs test. Second, the oxidase activity of ceruloplasmin is also inhibited by adding sodium azide to the system, thus eliminating the influence of ceruloplasmin on d-ROMs levels (Alberti et al., 2000). Moreover, it has been shown that females have lower levels of ceruloplasmin in the blood compared with males (Alberti et al., 2000), which further eliminates the influence of ceruloplasmin in our samples. Furthermore, the d-ROMs test has been validated by EPR spectrometry, which is considered as the golden standard method to measure oxidationreduction phenomena in vitro (Alberti et al., 2000). Moreover, the d-ROMs test has successfully been validated in large populations of healthy subjects, including athletes, and is regarded as a reproducible method for the quantitative evaluation of the peroxidation of organic compounds (Iamele et al., 2002; Bonina et al., 2005; Banfi et al., 2006). It has also been argued that the non-automated handling of the samples results in imprecision of d-ROMs (Iamele et al., 2002). However, in the present study, the analysis was fully automated. According to the manufacturer's instructions, a value between 301 and 320 CARR $U$ indicates a borderline condition of oxidative stress status, while a value above 320 CARR U indicates oxidative stress (Diacron Inter- national). In the present study, the average d-ROMs value after the soccer game was $249 \pm 25$ CARR U, which indicates that no peroxidation had occurred.

Previous studies in male players show increased lipid peroxidation (MDA and TBARS) in the plasma following soccer games (Ascensão et al., 2008; Ispirlidis et al., 2008) and a soccer-specific running protocol (Kingsley et al., 2005). The lipid peroxidation levels measured by MDA and TBARS were elevated up to $72 \mathrm{~h}$ following the soccer games (Ascensão et al., 2008; Ispirlidis et al., 2008) while hydroperoxide was normalized within $24 \mathrm{~h}$ after the soccerspecific running protocol (Kingsley et al., 2005). The lack of lipid peroxidation measured by d-ROMs in our results compared with previous studies may be due to several factors. First, the players in our study were well trained and had a high training volume. Previous studies have demonstrated that regular training is associated with a strengthening of the endogenous antioxidant defense system and thus well-trained athletes probably have improved protection against oxidative insults as compared with sedentary subjects (Brites et al., 1999). Second, differences between our results and previous studies may be due to the fact that all previous studies were performed in males. In this respect, there are indications that estrogen hormones have antioxidant functions (Kendall \& Eston, 2002) and may thus contribute to a more efficient antioxidant defense system in women compared with men. This is supported by a previous study in females showing that lipid peroxidation did not occur in response to exercise despite an increase in GSSG (Chung et al., 1999). Third, the observed increase in GSSG was paralleled by a robust antioxidant response. This indicates that free radical production was increased during the game, but was effectively quenched by a robust antioxidant defense system. This may explain the lack of significant increase in peroxidation measured by d-ROMs. Our results suggest a model where the concomitant increase in endogenous and dietary antioxidants in response to the transient increase in GSSG prevented the occurrence of lipid peroxidation measured by d-ROMs in well-trained female players (Fig. 2). It is also suggested that the antioxidant defense systems effectively quenched increased levels of ROS/RNS and hence inhibited excessive lipid peroxidation measured by d-ROMs.

GSSG levels returned to baseline $21 \mathrm{~h}$ after the soccer game in well-trained female players. This recovery pattern was paralleled by the recovery of the endogenous antioxidants (uric acid and TGSH). This suggests that the endogenous antioxidants are mainly involved in the early phase of the defense system against increased ROS/RNS. In contrast, uric acid was elevated for more than $72 \mathrm{~h}$ following games in male soccer players (Ascensão et al., 2008; Ispirli- 


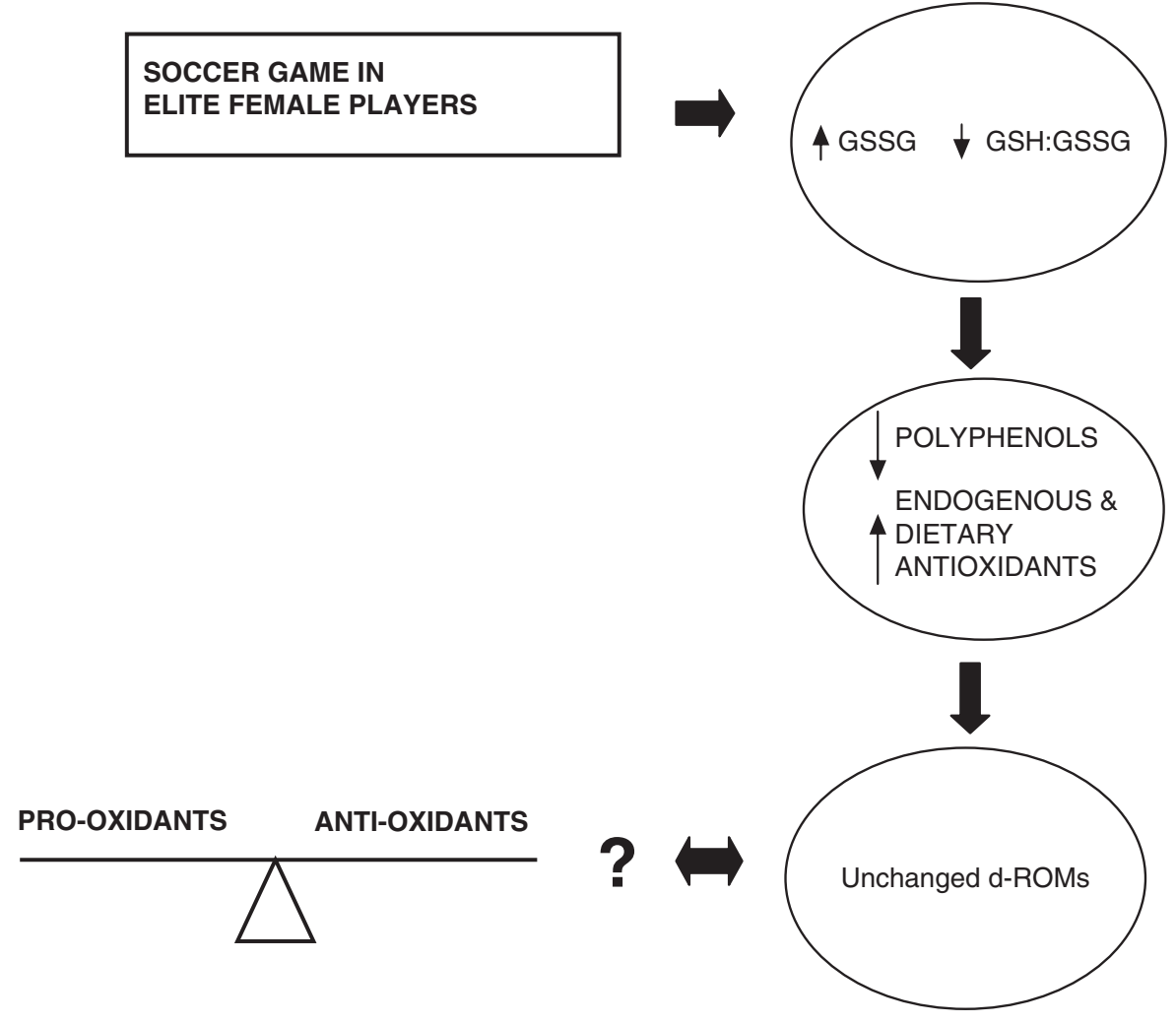

Fig. 2. Hypothetical model describing changes in oxidative stress markers and endogenous and dietary antioxidants following intermittent exercise in well-trained female soccer players.

dis et al., 2008). In the present study, one dietary antioxidant (AA) returned to baseline $21 \mathrm{~h}$ after the game while two others (total polyphenols and $\alpha$-tocopherol) did not. This suggests that the response of the dietary antioxidants may last longer than that of the endogenous antioxidants. The longterm reduction of polyphenols can also be explained by the fact that they are not stored in peripheral tissues and may only be available in the circulation (Rechner et al., 2002). As such, polyphenols may not be replaced in the circulation as fast as other antioxidant compounds. Interestingly, we observed an increase in several carotenoid compounds that occurred $21 \mathrm{~h}$ after the game. The delayed increase in carotenoids could be due to their slow release from their main storage site (adipose tissue) following the game. Our findings suggest that in well-trained female athletes, the carotenoid response can serve to strengthen the plasma antioxidant defense system following strenuous exercise.

In conclusion, our results indicate that intermittent exercise in well-trained female soccer players induces a transient increase in oxidized glutathione and a reduced GSH:GSSG ratio accompanied by a robust response of the endogenous and dietary antioxidant defense systems. The orchestrated endogenous and dietary antioxidant responses in well-trained female athletes helped to prevent the occurrence of lipid peroxidation measured by d-ROMs after a soccer game.

\section{Perspectives}

Antioxidant supplements are commonly used in soccer. Our results imply that female elite players have an effective and well-regulated antioxidant defense system. However, as our study has been performed on female players, our conclusion might not be applicable for male soccer. Nevertheless, based on our results, the use of antioxidant supplements in female soccer is questionable.

Key words: d-ROMs, glutathione, lipid peroxidation, polyphenols, intermittent exercise, endurance training.

\section{Acknowledgements}

This study was supported by grants from Swedish National Centre for Research in Sports $(89 / 06)$. The study was performed at Olympiatoppen in Norway. The authors would like to thank the players from KIF Örebro DFF and Kolbotn IL. 


\section{References}

Aguiló A, Tauler P, Fuentespina E, Tur J, Córdova A, Pons A. Antioxidant response to oxidative stress induced by exhaustive exercise. Physiol Behav 2005: 84: 1-7.

Alberti A, Bolognini L, Macciantelli D, Caratelli M. The radical cation of $\mathrm{N}$, N-Diethyl-para-phenylendiamine: a possible indicator of oxidative stress in biological samples. Res Chem Intermed 2000: 26: 253-267.

Andersson H, Raastad T, Nilsson J, Pausen G, Garthe I, Kadi F. Neuromuscular fatigue and recovery in elite female soccer: effects of active recovery. Med Sci Sports Exerc 2008: 40: 372-380.

Ascensão A, Rebelo A, Oliviera E, Marques F, Pereira L, Magalhães J. Biochemical impact of a soccer matchanalysis of oxidative stress and muscle damage markers throughout recovery. Clin Biochem 2008: 41: 841-851.

Banfi G, Malavazos A, Iorio E, Dolci A, Doneda L, Verna R, Corsi M. Plasma oxidative stress biomarkers, nitric oxide and heat shock protein 70 in trained elite soccer players. Eur J Appl Physiol 2006: 96: 483-486.

Bangsbo J. The physiology of soccer- with special reference to intense intermittent exercise. Acta Physiol Scand Suppl 1994: 619: 1-155.

Blomhoff R. Dietary antioxidants and cardiovascular disease. Curr Opin Lipidol 2005: 16: 47-54.

Bonina F, Puglia C, Cimino F, Trombetta D, Tringali G, Roccazzello A, Insirello E, Rapisarda P, Saija A. Oxidative stress in handball players: effect of supplementation with a red orange extract. Nutrit Res 2005: 25: 917-924.

Brites FD, Evelson PA, Christiansen MG, Nicol MF, Basílico MJ, Wikinski RW, Llesuy SF. Soccer players under regular training show oxidative stress but an improve plasma antioxidant status. Clin Sci Lond 1999: 96: 381-385.

Cesarone M, Belcaro G, Carratelli M, Cornelli U, De Sanctis M, Incandela L, Barsotti A, Terranova R, Nicolaides A. A simple test to monitor oxidative stress. Int Angiol 1999: 18: 127-130.

Chung S, Goldfarb A, Jamurtas A, Hegde $\mathrm{S}$, Lee J. Effect of exercise during the follicular and luteal phase on indices of oxidative stress in healthy women. Med Sci Sports Exerc 1999: 31: 409-413.

Davies KJ, Quintanilha A, Brooks G, Packer L. Free radicals and tissue damage produced by exercise. Biochim Biophys Res Commun 1982: 107: 1198-1205.

Djordjević V. Free radicals in cell biology. Int Rev Cytol 2004: 237: 57-89.
Erel O. A new automated colorimetric method for measuring total oxidant status. Clin Biochem 2005: 38: 1103-1111.

Halliwell B, Rafter J, Jenner A. Health promotion by flavonoids, tocopherols, tocotrienols, and other phenols: direct or indirect effects? Antioxidant or not? Am J Clin Nutr 2005: 81: 268S-276S.

Harma I, Harma M, Erel O. Measuring plasma oxidative stress biomarkers in sport medicine. Eur J Appl Physiol 2006: 97: 505.

Hellsten Y, Tullson P, Richter E, Bangsbo J. Oxidation of urate in human skeletal muscle during exercise. Free Radic Biol Med 1997: 22: 169-174.

Iamele L, Fiocchi R, Vernocchi A. Evaluation of an automated spectrophotometric assay for reactive oxygen metabolites in serum. Clin Chem Labor Med 2002: 40: 673-676.

Ispirlidis I, Fatouros I, Jamurtas A, Nikolaidis M, Michailidis I, Douroudos I, Margonis K, Chatzinikolaou A, Kalistratos E, Katrabasas I, Alexiou V, Taxildaris K. Time-course of changes in inflammatory and performance responses following a soccer game. Clin J Sport Med 2008: 18: 423-431.

Jackson MJ. Free radical mechanisms in exercise-related muscle damage. In: Reznick AZ, Packer L, Sen CK, Holloszy JO, Jackson MJ, eds. Oxidative stress in skeletal muscle. Basel: Birkhauser Verlag, 1998: 75-86.

Ji LL. Free radicals and antioxidants in exercise and sports. In: Garrett W, Kirkendall D, eds. Exercise and sport science. Philadelphia: Lippincott Williams \& Wilkins, 2000: 299-317.

Ji LL. Antioxidants and oxidative stress in exercise. Exp. Biol. Med. 1999: 222: 283-292.

Karlsen A, Blomhoff R, Gundersen T. High-throughput analysis of vitamin $\mathrm{C}$ in human plasma with the use of HPLC with monolithic column and UVdetection. J Chromatogr B Analyt Technol Biomed Life Sci 2005: 824: 132-138.

Karlsen A, Blomhoff R, Gundersen T. Stability of whole blood and plasma ascorbic acid. Eur J Clin Nutr 2007: 61: 1233-1236.

Kendall B, Eston R. Exercise-induced muscle damage and potential protective role of estrogens. Sports Med 2002: 32: 103-123.

Kingsley M, Wadsworth D, Kilduff L, McEneny J, Benton D. Effects of phosphatidylserine on oxidative stress following intermittent running. Med Sci Sports Exerc 2005: 37: 1300-1306.
Krustrup P, Mohr M, Ellingsgaard H, Bangsbo J. Physical demands during an elite female soccer game: importance of training status. Med Sci Sports Exerc 2005: 37: 1242-1248.

Leeuwenburgh C, Ji L. Alteration of glutathione and antioxidant status in unfed and refed rats. J Nutrit 1996: 126: 1833-1843.

Maskarinec G, Chan C, Meng L, Franke A, Cooney R. Exploring the feasibility and effects of a high-fruit and vegetable diet in healthy women. Cancer Epidemiol Biomarkers Prev 1999: 8: 919-924.

Maughan R, Burke L, Coyle E, eds. Food, nutrition and sports performance II. The international olympic committee consensus on sports nutrition. New York: Routledge, 2004.

Morillas-Ruiz J, Villegas García JA, López FJ, Vidal-Guevara ML, Zafrilla P. Effects of polyphenolic antioxidants on exercise-induced oxidative stress. Clin Nutr 2006: 25: 444-453.

Nakayama K, Terawaki H, Nakayama M, Iwabuchi M, Sato T, Ito S. Reduction of serum antioxidative capacity during hemodialysis. Clin Exp Nephrol 2007: 11: 218-224.

Powers S, Jackson M. Exercise-induced oxidative stress: mechanisms and impact on muscle force production. Physiol Rev 2008: 88: 1243-1276.

Rechner AR, Kuhnle G, Bremer P, Hubbard GP, Moore KP, Rice-Evans C. The metabolic fate of dietary polyphenols in humans. Free Radic Biol Med 2002: 33: 220-235.

Richheimer S, Kent M, Bernart M. Reversed-phase high-performance liquid chromatographic method using a pentafluorophenyl bonded phase for analysis of tocopherols. J Chrom A 1994: 677: 75-80.

Sakhi AK, Russnes KM, Smeland S, Blomhoff R, Gundersen TE. Simultaneous quantification of reduced and oxidized glutathione in plasma using a two-dimensional chromatographic system with parallel porous graphitized carbon columns coupled with fluorescence and Coulometric electrochemical detection. $\mathbf{J}$ Chromatogr 2006: 1104: 179-189.

Saltin B, Åstrand P. Maximal oxygen uptake in athletes. J Appl Physiol 1967: 23: 353-358.

Sastre J, Asensi M, Gasgo E, Pallardo F, Ferrero J, Furukawa T, Vina J. Exhaustive physical exercise causes oxidation of glutathione status in blood: prevention by antioxidant administration. Am J Physiol 1992: 263: R992-R995. 


\section{Soccer, anti-oxidants and oxidative stress}

Sen C, Marin E, Kretzschmar M, Hänninen O. Skeletal muscle and liver glutathione homeostasis in response to training, exercise and immobilisation. J Appl Physiol 1992: 73: 1265-1272.

Sen CK, Packer L. Thiol homeostasis and supplements in physical exercise. Am J Clin Nutr 2000: 72: 653S-669S.

Tauler P, Aguiló A, Guix P, Jiménez F, Villa G, Tur JA, Córdova A, Pons A. Pre-exercise antioxidant enzyme activities determine the antioxidant enzyme erythrocyte response to exercise. J Sports Sci 2005: 23: 5-13.

Tauler P, Ferrer M, Sureda A, Pujol P, Drobnic F, Tur JA, Pons A.
Supplementation with an antioxidant cocktail containing coenzyme Q prevents plasma oxidative damage induced by soccer. Eur $\mathbf{J}$ Appl Physiol 2008: 104: 777-785.

Thompson D, Williams C, Kingsley M, Nicholas C, Lakomy H, McArdle F, Jackson M. Muscle soreness and damage parameters after prolonged intermittent shuttle-running following acute vitamin $\mathrm{C}$ supplementation. Int J Sports Med 2001: 22: 68-75.

Viguie CA, Frei B, Shigenaga MK, Ames BN, Packer L, Brooks GA. Antioxidant and indexes of oxidative stress during consecutive days of exercise. J Appl Physiol 1993: 75: 566-572.

Watson T, Callister R, Taylor R, Sibbritt D, MacDonald-Wicks L, Garg M. Antioxidant restriction and oxidative stress in short-duration exhaustive exercise. Med Sci Sports Exerc 2005: 37: 63-71.

Wayner D, Burton G, Ingold K, Barclay $\mathrm{L}$, Locke $\mathrm{S}$. The relative contributions of vitamin $\mathrm{E}$, urate, ascorbate and proteins to the total peroxyl radicaltrapping antioxidant activity of human blood plasma. Biochim Biophys Acta 1987: 924: 408-419. 